ORIGINAL ARTICLE

\title{
The influence of extent of surgical margin positivity on prostate specific antigen recurrence
}

\author{
R E Emerson, M O Koch, T D Jones, J K Daggy, B E Juliar, L Cheng
}

J Clin Pathol 2005;58:1028-1032. doi: 10.1136/icp.2005.025882

See end of article for authors' affiliations

\section{Correspondence to:}

Dr L Cheng, Department of Pathology and Laboratory Medicine, Indiana

University Medical Center, University Hospital 3465, 550 North University Blvd, Indianapolis, IN 46202 USA; Icheng@iupui.edu

Accepted for publication 22 February 2005
Background: Positive surgical margins are an adverse prognostic factor in patients undergoing prostatectomy for prostate cancer. The extent of margin positivity varies and its influence on clinical outcome is uncertain.

Aims: To evaluate the linear extent of margin positivity and the number and location of positive sites as prognostic indicators in a series of prostatectomy specimens evaluated with the whole mount technique. Methods: Eighty six consecutive margin positive prostatectomy specimens were evaluated, and all pathology data were collected prospectively. The linear extent of margin positivity was measured with an ocular micrometer and the total extent of all positive sites was summed. The total number of sites with positive margins and anatomical sites of the positive margins were analysed.

Results: The linear extent of margin positivity ranged from 0.01 to $68 \mathrm{~mm}$ (mean, 6.8; median, 3.0) and was associated with prostate specific antigen (PSA) recurrence in univariate logistic regression $(p=0.031)$. In addition, the extent of margin positivity weakly correlated with preoperative PSA $(p=0.017)$ and tumour volume $(p=0.013)$, but not with age, prostate weight, Gleason score, pathological stage, or perineural invasion. The total number of positive sites was significantly higher in patients with PSA recurrence $(p=0.037)$. The location of the positive margin site was not associated with PSA recurrence. The extent of margin positivity correlated with PSA recurrence in univariate analysis, although it had only marginal predictive value when adjusted for Gleason score $(p=0.076)$.

Conclusions: The extent of margin positivity correlates with PSA recurrence in univariate analysis, although it has no predictive value independent of Gleason score.
L ocal recurrence and distant metastasis remain difficult to predict after prostatectomy, but several prognostic indiLators including margin involvement have been identified. ${ }^{1-9}$ Positive margins are seen in $12-43 \%$ of prostatectomy specimens. $^{2710-13}$ The causes and clinical consequences of positive prostatectomy margins have been discussed extensively. ${ }^{4} 791214$ Margin status is related to other pathological variables, such as maximum tumour diameter, number of blocks involved by tumour, and percentage of cancer in biopsy specimens, which are indicators of tumour volume. ${ }^{5}$ 15-19 Although disease recurrence is more common in patients with surgical margin involvement, not all patients with microscopically involved surgical margins experience recurrence. ${ }^{2} 3$ 5-7 20

"Local recurrence and distant metastasis remain difficult to
predict after prostatectomy, but several prognostic indica-
tors including margin involvement have been identified"

We investigated the relation between the linear extent of surgical margin involvement and the likelihood of prostate specific antigen (PSA) recurrence in a series of patients with positive surgical margins in their radical prostatectomy specimens. The numbers of involved sites and the locations of the involved sites were also evaluated.

\section{MATERIALS AND METHODS}

The group of patients studied was taken from a series of 369 consecutive patients treated by radical retropubic prostatectomy, with or without bilateral pelvic lymph node dissection, for adenocarcinoma of the prostate at Indiana University Hospital, Indianapolis, USA from 1999 to 2003. From this series of 369 cases, 86 cases $(23 \%)$ had positive margins. None of the patients had preoperative radiotherapy or androgen deprivation treatment. Preoperative PSA was measured with the Immulite DPC chemiluminescent assay (Diagnostics Products, Los Angeles, California, USA). Only patients without evidence of distant metastasis were included.

The prostatectomy specimens were processed by the whole mount technique as described previously. ${ }^{16} 21-26$ Specimens were weighed, measured, inked, and fixed in 10\% formalin. The base and apex were removed and sectioned at 3-5 mm intervals and the seminal vesicles were removed and sectioned. The specimens were then sectioned perpendicular to the long axis, submitted for processing in large cassettes, and entirely embedded. Five micron sections were then prepared and stained with haematoxylin and eosin.

All pathological data, including the extent of margin positivity and location of positive margin site, were collected prospectively by a single genitourinary pathologist (LC). Margin involvement was defined as the presence of malignant cells touching the inked margin (fig l). ${ }^{15}$ The sites of margin involvement were marked on the slides. The numbers and locations of the sites of margin involvement were recorded. Location was designated as seminal vesicle, apex, bladder base, right anterior, left anterior, right posterior, and left posterior. For analytical purposes, right and left anterior and right and left posterior were combined, and designated as lateral. The linear extent of margin involvement was measured at each involved site with an ocular micrometer. For specimens with multiple involved sites, the measurements were summed. The tumour volume was calculated using the grid method. ${ }^{16}{ }^{27}$ Pathological stage was assessed according to the 1997 TNM criteria ${ }^{28}$ and tumours were classified as either $\mathrm{T} 2$ of $\mathrm{T} 3$ for purposes of statistical

Abbreviations: OR, odds ratio; PSA, prostate specific antigen 


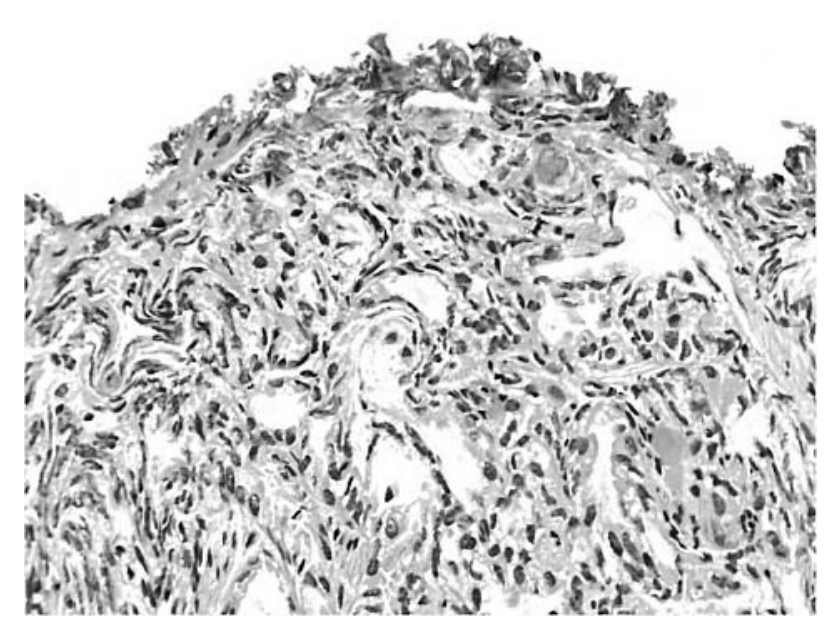

Figure 1 Involvement of surgical margin by prostatic adenocarcinoma. A positive margin was defined as the presence of malignant cells touching the inked margin.

analysis. The 1997 TNM criteria were used rather than the 2002 criteria because of the extreme rarity of 2002 pT2b tumours. In our previous study, we found that a true pT2b tumour probably does not exist. ${ }^{29}$ The Gleason score was determined according to the Gleason grading system, ${ }^{30}$ and was grouped as 6 or less, 7 , or 8 or more for statistical analysis.

The patients were followed at three month intervals during the first year and at six month intervals during the second year. Thereafter, follow up was adjusted according to the clinical situation but was obtained at least annually. The median length of follow up was 12 months (range, 1.5-48). A

Table 1 Clinical and pathological findings in 86 margin positive patients

\begin{tabular}{lc}
\hline Characteristics and measures ( $\mathbf{n}=86)$ & $\mathbf{N}(\%)$ or median (range) \\
\hline Age & $62(45-76)$ \\
Preoperative PSA (ng/ml) & $7.4(3-150)$ \\
Prostate weight (g) & $38(18-149)$ \\
Findings of radical prostatectomy specimen & \\
Primary Gleason grade (G1) & \\
2 & $5(5.8 \%)$ \\
3 & $56(65.1 \%)$ \\
4 & $21(24.4 \%)$ \\
5 & $4(4.7 \%)$ \\
Secondary Gleason grade (G2) & $8(9.3 \%)$ \\
2 & $34(39.5 \%)$ \\
3 & $36(41.9 \%)$ \\
4 & $8(9.3 \%)$ \\
5 & $13(15.1 \%)$ \\
Gleason score & $15(17.4 \%)$ \\
5 & $46(53.5 \%)$ \\
6 & $1(1.2 \%)$ \\
7 & $11(12.8 \%)$ \\
8 & $3(3.5 \%)$ \\
9 & $44(51.2 \%)$ \\
Pathological stage (TNM 1997) & $30(34.9 \%)$ \\
T2a & $9(10.5 \%)$ \\
T2b & $3.1(0.4-38)$ \\
T3a & $2(2.3 \%)$ \\
T3b & $38(44.2 \%)$ \\
Tumour volume (cc) & $10(11.6 \%)$ \\
Lymph node metastasis (positive) & $77(89.5 \%)$ \\
Extraprostatic extension (positive) & $85(98.8 \%)$ \\
Seminal vesicle invasion (positive) & $77(89.5 \%)$ \\
Perineural invasion (positive) & $22(25.6 \%)$ \\
High grade PIN (positive) & \\
Multifocality of cancer (positive) & \\
PSA recurrence & prostate specific antigen. \\
\hline PIN, prostate intraepithelial neoplasia; PSA \\
\end{tabular}

serum PSA concentration of at least $0.1 \mathrm{ng} / \mathrm{ml}$ was used to define PSA recurrence. ${ }^{9} 3132$

Univariate and multiple logistic regression were used to test the effect of predictors on PSA recurrence. Because there were only 22 subjects with PSA recurrence, limiting the number of predictors to a maximum of two, a series of multiple regressions was performed with two predictors in each analysis. Fisher's exact test was used to test the effect of location on PSA recurrence. Comparison of the extent of margin by tumour stage and univariate analysis of the number of involved sites were performed using Wilcoxon non-parametric tests. Correlations were evaluated using Spearman correlation coefficients. Tests were conducted as two sided with marginal significance at $\alpha=0.10$ and significance at $\alpha=0.05$. Statistical analysis was performed using SAS software V8.2 (Cary, North Carolina, USA).

\section{RESULTS}

From the series of 369 patients, 86 prostatectomy specimens (23\%) had positive margins. Table 1 lists the clinical and pathological findings in these patients. PSA recurrence was seen in 22 of these patients.

Established pathological features were predictive for PSA recurrence (table 2). These included tumour stage $(\mathrm{p}=0.050 ;$ odds ratio $(\mathrm{OR}), 0.366)$, Gleason score $(\mathrm{p}=0.002 ; \mathrm{OR}, 2.286)$, and tumour volume (measured in $\mathrm{cm}^{3} ; \mathrm{p}=0.013 ;$ OR, 1.222).

The extent of margin positivity ranged from 0.01 to $68 \mathrm{~mm}$ (mean, 6.76; median 3.0). The extent of margin positivity was associated with PSA recurrence $(\mathrm{p}=0.031$; OR, 1.065). The extent of margin positivity was weakly associated with other prognostic indicators, including preoperative PSA $(r=0.26$; $\mathrm{p}=0.017)$ and tumour volume $(r=0.27 ; \mathrm{p}=0.013)$. The extent of margin positivity did not correlate with Gleason score or tumour stage.

Univariate and multiple logistic regression analyses were performed considering the effect of the extent of margin positivity, tumour stage, Gleason score, and tumour volume on PSA recurrence. All predictors had predictive value in univariate analyses (table 2). Because the Gleason score was the strongest univariate predictor, the effect of each predictor adjusted for the Gleason score was analysed in a series of two predictor multiple regressions. The Gleason score tested separately with the predictors (1) tumour stage, (2) tumour volume, and (3) the extent of positivity were consistently significant in predicting PSA recurrence $(p=0.005$, $p=0.007$, and $p=0.005$, respectively). Adjusted for Gleason score, increased tumour volume and margin extent were marginally significant and tumour stage was not a significant predictor of PSA recurrence $(p=0.075$, $\mathrm{p}=0.076$, and $\mathrm{p}=0.336$, respectively; table 3 ).

The number of positive margin sites was also associated with PSA recurrence $(p=0.037)$. The patients without PSA recurrence had a mean of 1.80 (SD, 0.99) positive sites, whereas those with recurrence had a mean of 2.45 (SD, 1.41) positive sites. When only the number of lateral sites was considered, there was a marginal association with PSA recurrence $(p=0.064)$. The cases without PSA recurrence

Table 2 Univariate predictors of prostate specific antigen recurrence

\begin{tabular}{lll}
\hline Characteristics and measures $(\mathbf{n}=\mathbf{8 6})$ & $\mathbf{p ~ V a l u e}$ & Odds ratio \\
\hline Tumour stage (T2 $v$ T3) & 0.050 & 0.366 \\
Gleason score & 0.002 & 2.286 \\
Tumour volume (measured in $\mathrm{cm}^{3}$ ) & 0.013 & 1.222 \\
Extent of margin positivity & 0.031 & 1.065 \\
\hline
\end{tabular}


Table 3 Predictors of prostate specific antigen recurrence adjusted for Gleason score

\begin{tabular}{llll}
\hline Characteristics and measures $(\mathbf{n}=\mathbf{8 6})$ & $\mathbf{p}$ Value & Odds ratio & $\begin{array}{l}\text { Gleason score } \mathbf{p} \\
\text { value }\end{array}$ \\
\hline Tumour stage (T2 $\mathbf{v}$ T3) & 0.336 & 0.584 & 0.005 \\
Tumour volume (measured in $\mathrm{cm}^{3}$ ) & 0.075 & 1.163 & 0.007 \\
Extent of margin positivity & 0.076 & 1.047 & 0.005 \\
Number of positive sites & 0.038 & 1.627 & 0.002 \\
\hline
\end{tabular}

had a mean of 1.25 (SD 0.82) positive sites, whereas those with recurrence had a mean of 1.77 (SD 1.31) positive sites. In multiple logistic regression analysis adjusted for Gleason score $(p=0.002)$, a greater number of positive sites was associated with PSA recurrence $(\mathrm{p}=0.038$; table 3$)$.

The site of margin positivity was evaluated for cases with apex, lateral, bladder base, and seminal vesicle involvement with or without involvement of other sites (table 4). The site was not significant as a predictor of PSA recurrence. Site was also not significant when cases with apex involvement only were evaluated ( $p=0.437)$.

\section{DISCUSSION}

Our results from a series of patients with positive margins indicated that the linear extent of margin involvement in prostatectomy specimens adjusted for Gleason score had no significance as a predictor of disease recurrence. In univariate analysis, patients with a greater linear extent of margin involvement were more likely to experience recurrence. However, in separate models adjusted for Gleason score, margin extent, tumour stage, and tumour volume were not significant predictors. Only Gleason score was significantly predictive of PSA recurrence in univariate and two predictor models. The extent of margin involvement was weakly correlated with preoperative PSA and tumour volume.

These findings are not unexpected given the less than absolute relation between surgical margin status and PSA recurrence in patients followed over a relatively long time period..$^{23-720}$ Although recurrence may be more frequent in patients with positive margins, more than one third of patients with positive margins will remain recurrence free at five years, ${ }^{2}$ and two thirds of patients with only focal margin involvement will remain disease free at five years. ${ }^{7}$ Conversely, margin negative, extracapsular extension negative patients may have relapse rates as high as $40 \%$ at three years if other prognostic factors are unfavourable. ${ }^{33}$ In fact, survival curves for margin positive patients are similar to those for extracapsular extension positive, margin negative patients. ${ }^{356}$ Although in some studies margin status has been more important than extraprostatic extension of carcinoma in the prediction of recurrence, ${ }^{12}{ }^{13}$ other studies have found limited impact on survival adjusted for extraprostatic extension and seminal vesicle invasion, ${ }^{5}$ and this is reflected in the AJCC staging system. ${ }^{12}{ }^{34}$ Because the completeness of surgical removal of the prostatic capsule is variable, a single tumour might be classified as T3 with negative margins if the capsule is removed intact or as T2 with positive margins if the capsule is not removed intact. This situation may result in a similar prognosis for margin positive and extracapsular extension positive, margin negative patients. In one study of patients with positive margins, a Gleason score of 7, and 10 years of follow up, biochemical (PSA) recurrence was seen in 52\% of patients but local recurrence could be documented in only $6 \%$ of patients. ${ }^{20}$

It is difficult to explain why patients with positive margins do not display local recurrence in a more predictable manner. Some patients with surgical margins that histologically appear positive may have no residual viable tumour after local tissue destruction as a result of the effects of disrupted vasculature, cautery, and inflammation related to surgery. One study of patients with positive margins in the region of the neurovascular bundle demonstrated residual carcinoma in only six of 10 patients upon repeat excision. ${ }^{35}$

The linear extent of margin positivity has been studied previously. Epstein et al showed significant differences for negative, focally positive (diagnosed when "the involved site(s) were limited and present in only one or two areas") and extensively positive margin patients. ${ }^{13}$ The percentages of recurrence free patients at five years were significantly different in univariate analysis: $87 \%$ for margin negative patients, $60 \%$ for focally positive margin patients, and 35\% for extensively margin positive patients. ${ }^{13}$ Epstein et al later demonstrated that this relation was also true for the subgroup of seminal vesicle positive patients. ${ }^{36}$ Neither of

\begin{tabular}{|c|c|c|c|}
\hline \multirow[b]{2}{*}{ Margin involved } & \multicolumn{2}{|c|}{ PSA recurrence } & \multirow[b]{2}{*}{$\mathrm{p}$ Value } \\
\hline & No & Yes & \\
\hline \multicolumn{4}{|l|}{ Apex } \\
\hline No & 38 (75\%) & $13(26 \%)$ & $>0.999$ \\
\hline Yes & $26(74 \%)$ & $9(26 \%)$ & \\
\hline \multicolumn{4}{|l|}{ Lateral $^{*}$} \\
\hline No & $9(69 \%)$ & $4(31 \%)$ & 0.732 \\
\hline Yes & $55(75 \%)$ & $18(25 \%)$ & \\
\hline \multicolumn{4}{|l|}{ Bladder base } \\
\hline No & 55 (76\%) & $17(24 \%)$ & 0.336 \\
\hline Yes & $9(64 \%)$ & $5(36 \%)$ & \\
\hline \multicolumn{4}{|l|}{ Seminal vesicle } \\
\hline No & $63(75 \%)$ & $21(25 \%)$ & 0.448 \\
\hline Yes & $1(50 \%)$ & $1(50 \%)$ & \\
\hline
\end{tabular}


these studies reported the significance of the extent of margin positivity for margin positive patients in multiple regression analysis. ${ }^{13} 37$

Babaian et al similarly found strikingly different rates of biochemical relapse beyond five years in patients with more or less than $3 \mathrm{~mm}$ of margin involvement. ${ }^{31}$ Margin positive patients with a length of margin involvement of $3 \mathrm{~mm}$ or less showed an overall recurrence rate of $14 \%$, whereas patients with greater than $3 \mathrm{~mm}$ of margin involvement had an overall recurrence rate of $53 \%{ }^{31}$ In fact, in margin positive patients with pT2 disease, the extent of margin positivity was the only predictor of recurrence in multiple regression analysis, and for the group of patients with seminal vesicle invasion it was one of three significant predictors. ${ }^{31}$ Similarly, using $3 \mathrm{~mm}$ as the division between focal and extensive margin involvement, Weldon et al, with 200 patients and a mean follow up of 35 months, found PSA recurrence in $0 \%$ of margin negative patients, $26 \%$ of focally margin positive patients, and $69 \%$ of extensively margin positive patients. ${ }^{38}$ This difference was highly significant in univariate analysis, but adjustment for other predictors was not performed. ${ }^{38}$

In contrast, Watson et al found that focal margin involvement, defined as involvement of only one gland in one section, was associated with a $31 \%$ chance of recurrence, whereas greater than focal involvement was associated with a $38 \%$ chance of recurrence. ${ }^{7}$ In contrast to the previous studies and our findings, this difference was not significant, even in univariate analysis. ${ }^{7}$

The number of positive sites was significant in univariate and in multiple logistic regression analysis adjusted for Gleason score. The number of sites is probably a correlate of tumour volume and has previously been shown to be significant as a predictor of recurrence in univariate analyses in two studies. ${ }^{71}$ Freedom from progression was seen in $71 \%$ of patients with a single positive margin and $52 \%$ of patients with involvement of more than one margin in one study, ${ }^{7}$ and in $77 \%$ of patients with a single positive margin and $68 \%$ of patients with involvement of more than one margin in the second. ${ }^{11}$ Another study, reported in abstract form, showed absence of progression at five years in $71 \%$ of patients with involvement of a single site and $36 \%$ of patients with involvement of three or more sites. ${ }^{39}$

\section{"We do not advocate reporting the extent of margin involvement as a standard part of the surgical pathology report on prostatectomy specimens"}

We found no significant differences in PSA recurrence rates based on the site of the positive margin, but our sample size is smaller than that of some previous studies investigating this variable. Blute et al, in a series of 697 margin positive patients, found a significantly lower recurrence free survival rate in patients with margin involvement at the prostate base $(58 \%)$ compared with the anterior surface, posterior surface, or apex $(78-79 \%)$ in analysis adjusted for tumour grade and preoperative PSA. $^{11}$ Obek et al, in a series of 151 margin positive patients, found significantly higher recurrence rates in patients with positive bladder neck and posterolateral margins than in patients with positive margins at other sites. ${ }^{40}$ The relative risk for recurrence was 3.25 for patients with positive bladder neck margins and 2.81 for patients with positive posterolateral margins compared with those with positive margins as a whole..$^{40}$ Another study, reported in abstract form, also reported significant differences in recurrence rates with five year progression free survival for patients with a single involved margin: $69 \%$ for an apical margin, $27 \%$ for a base margin, $48 \%$ for a posterior margin, and $79 \%$ for an anterior margin. ${ }^{39}$ This study included 169
Take home messages

- The linear extent of surgical margin involvement was associated with prostate specific antigen (PSA) recurrence after prostatectomy in univariate analysis

- The linear extent of surgical margin positivity was associated with other standard pathological variables predictive of cancer recurrence including preoperative PSA and tumour volume

- It was not a significant predictor of PSA recurrence in multiple logistic regression analysis

margin positive patients. A study with 73 margin positive patients, similar in size to our study, failed to demonstrate significant differences in progression free survival based on the site of the positive margin. ${ }^{7}$

Because only a small number of patients were studied with involvement of a given site, it is probable that our study also lacks the statistical power necessary to detect differences in recurrence rates based on the site of margin positivity. Sample size was also insufficient for multiple logistic regression with more than two predictors, and larger studies with greater power might clarify the effect of some factors. Some other limitations of this study are apparent. Follow up may not be of adequate length to identify all patients who will eventually experience recurrence.

In summary, in univariate analysis the linear extent of surgical margin involvement was associated with PSA recurrence after prostatectomy. It was associated with other standard pathological variables predictive of cancer recurrence, including preoperative PSA and tumour volume. It was not a significant predictor of PSA recurrence in multiple logistic regression analysis and, therefore, we do not advocate reporting the extent of margin involvement as a standard part of the surgical pathology report on prostatectomy specimens.

\section{Authors' affiliations}

R E Emerson, T D Jones, L Cheng, Department of Pathology and Laboratory Medicine, Indiana University School of Medicine, Indianapolis, Indiana, IN 46202, USA

M O Koch, Department of Urology, Indiana University School of Medicine

J K Daggy, B E Juliar, Division of Biostatistics, Indiana University School of Medicine

\section{REFERENCES}

1 Cheng L, Darson MF, Bergstralh EJ, et al. Correlation of margin status and extraprostatic extension with progression of prostate carcinoma. Cancer 1999;86:1775-82.

2 Kupelian P, Katcher J, Levin $\mathrm{H}$, et al. Correlation of clinical and pathologic factors with rising prostate-specific antigen profiles after radical prostatectomy alone for clinically localized prostate cancer. Urology 1996;48:249-60.

3 D'Amico AV, Whittington R, Malkowicz SB, et al. Prostate specific antigen outcome based on the extent of extracapsular extension and margin status in patients with seminal vesicle negative prostate carcinoma of Gleason score $<$ or $=7$. Cancer 2000;88:2110-15.

4 Weider JA, Soloway MS. Incidence, etiology, location, prevention and treatment of positive surgical margins after radical prostatectomy for prostate cancer. J Urol 1998;160:299-315.

5 Ohori M, Wheeler TM, Kattan MW, et al. Prognostic significance of positive surgical margins in radical prostatectomy specimens. J Urol 1995; 154:1818-24.

6 Paulson DF, Walther PJ. Is grade or stage of primary importance in determining the outcome after radical prostatectomy for disease clinically confined to the prostate? Br J Urol 1989;63:301-5

7 Watson RB, Civantos F, Soloway MS. Positive surgical margins with radical prostatectomy: detailed pathological analysis and prognosis. Urology 1996;48:80-90.

8 Paulson DF. Impact of radical prostatectomy in the management of clinically localized disease. J Urol 1994;152:1826-30. 
9 van den Ouden D, Bentvelsen FM, Boeve ER, et al. Positive margins after radical prostatectomy: correlation with local recurrence and distant progression. Br J Urol 1993;72:489-94.

10 Zincke H, Bergstralh EJ, Blute ML, et al. Radical prostatectomy for clinically localized prostate cancer: long-term results of 1,143 patients from a single institution. J Clin Oncol 1994;12:2254-63.

11 Blute ML, Bostwick DG, Bergstralh EJ, et al. Anatomic site-specific positive margins in organ-confined prostate cancer and its impact on outcome after radical prostatectomy. Urology 1997:50:733-9.

12 Blute ML, Bostwick DG, Seay TM, et al. Pathologic classification of prostate carcinoma: the impact of margin status. Cancer 1998;82:902-8

13 Epstein JI, Pizov G, Walsh PC. Correlation of pathologic findings with progression after radical retropubic prostatectomy. Cancer 1993:71:3582-93.

14 Epstein $\mathrm{JI}$. Incidence and significance of positive margins in radical prostatectomy specimens. Urol Clin North Am 1996;23:651-63.

15 Cheng L, Slezak J, Bergstralh EJ, et al. Preoperative prediction of surgical margin status in patients with prostate cancer treated by radical prostatectomy. J Clin Oncol 2000;18:2862-8.

16 Eichelberger LE, Koch MO, Daggy JK, et al. Predicting tumor volume in radical prostatectomy specimens from patients with prostate cancer. Am J Clin Pathol 2003;120:386-91.

17 McNeal JE, Villers AA, Redwine EA, et al. Capsular penetration in prostate cancer: significance for natural history and treatment. Am J Surg Pathol 1990;14:240-7.

18 Jones EC. Resection margin status in radical retropubic prostatectomy specimens: relationship to type of operation, tumor size, tumor grade and local tumor extension. J Urol 1990;144:89-93.

19 Epstein Jl, Carmichael M, Partin AW, et al. Is tumor volume an independent predictor of progression following radical prostatectomy? A multivariate analysis of 185 clinical stage $B$ adenocarcinomas of the prostate with 5 years of follow up. J Urol 1993; 149:1478-81.

20 Han $M$, Pound CR, Potter SR, et al. Isolated local recurrence is rare after radical prostatectomy in men with Gleason 7 prostate cancer and positive surgical margins: therapeutic implications. J Urol 2001;165:864-6.

21 Poulos CK, Daggy JK, Cheng L. Prostate needle biopsies: multiple variables are predictive of final tumor volume in radical prostatectomy specimens. Cancer 2004;101:527-32.

22 Poulos CK, Koch MO, Eble JN, et al. Bladder neck invasion is an independent predictor of prostate-specific antigen recurrence. Cancer 2004;101:1563-8.

23 Hong H, Koch MO, Foster RS, et al. Anatomic distribution of periprostatic adipose tissue: a mapping study of 100 radical prostatectomy specimens. Cancer 2003:97:1639-43.

24 Cheng L, Koch MO, Daggy JK, et al. The combined percentage of Gleason pattern 4 and 5 is the best predictor of cancer progression after radical prostatectomy. J Clin Oncol 2005;23:2911-17.
25 Emerson RE, Koch MO Daggy J, et al. Closest distance between tumor and resection margin in radical prostatectomy specimens: lack of prognostic significance. Am J Surg Pathol 2005;29:224-9.

$26 \mathrm{Ng} \mathrm{JC}$, Koch MO, Daggy JK, et al. Perineural invasion in radical prostatectomy specimens: lack of prognostic significance. J Urol 2004; 172:2249-51.

27 Humphrey PA, Vollmer RT. Intraglandular tumor extent and prognosis in prostatic carcinoma: application of a grid method to prostatectomy specimens. Hum Pathol 1990;21:799-804.

28 Fleming ID, Cooper JS, Henson DE, et al. AJCC cancer staging manual. Philadelphia: Raven and Lippincott, 1997.

29 Eichelberger LE, Cheng L. Does pT2b prostate carcinoma exist? Critical appraisal of the 2002 TNM classification of prostate carcinoma. Cancer 2004; 100:2573-6

30 Gleason DF, Mellinger GT. Prediction of prognosis for prostatic adenocarcinoma by combined histologic grading and clinical stage. J Urol 1974;111:58-64.

31 Babaian RJ, Troncoso P, Bhadkamkar VA, et al. Analysis of clinicopathologic factors predicting outcome after radical prostatectomy. Cancer 2001;91:1414-22.

32 Koch MO, Foster RS, Bell B, et al. Characterization and predictors of prostate specific antigen progression rates after radical retropubic prostatectomy. J Urol 2000;164:749-53.

33 D'Amico AV, Whittington R, Malkowicz SB, et al. Prostate-specific antigen failure despite pathologically organ-confined and margin negative prostate cancer: the basis for an adjuvant therapy trial. J Clin Oncol 1997;15:1465-9.

34 Greene FL, Page DL, Flemming ID, et al. AJCC cancer staging manual, 6th ed. New York: Springer-Verlag, 2002.

35 Epstein Jl. Evaluation of radical prostatectomy capsular margins of resection: the significance of margins designated as negative, closely approaching, and positive. Am J Surg Pathol 1990; 14:626-32.

36 Epstein JI, Partin AW, Potter SR, et al. Adenocarcinoma of the prostate invading the seminal vesicle: prognostic stratification based on pathologic parameters. Urology 2000;56:283-8.

37 Epstein JI, Carmichael M, Walsh PC. Adenocarcinoma of the prostate invading the seminal vesicle: definition and relation of tumor volume, grade and margins of resection to prognosis. J Urol 1993;149:1040-5.

38 Weldon VE, Tavel FR, Neuwirth $\mathrm{H}$, et al. Patterns of positive specimen margins and detectable prostate specific antigen after radical perineal prostatectomy. J Urol 1995; 153:1565-9.

39 Gorbonos A, Ohori M, Maru H, et al. Prognostic significance of the site of the positive margin (+SM) in radical prostatectomy (RP) specimens. J Urol 2003;169:457.

40 Obek C, Sadek S, Lai S, et al. Positive surgical margins with radical retropubic prostatectomy: anatomic site-specific pathologic analysis and impact on prognosis. Urology 1999;54:682-8. 